\title{
Experimental and computational investigations of the effect of the electrode gap on capacitively coupled radio frequency oxygen discharges
}

Cite as: Phys. Plasmas 26, 013503 (2019); doi: 10.1063/1.5063543

Submitted: 28 September 2018 - Accepted: 20 November 2018 • Published Online:

07 January 2019

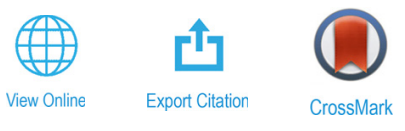

K. H. You, J. Schulze, ${ }^{2,3}$ A. Derzsi, ${ }^{2,4}$ Z. Donkó, $^{4}$ (D) H. J. Yeom, ${ }^{1,5}$ J. H. Kim, D. J. Seong, and Hyo-Chang Lee, a) (D)

\begin{abstract}
AFFILIATIONS
${ }^{1}$ Advanced Instrumentation Institute, Korea Research Institute of Standards and Science, Daejeon 34113, South Korea

${ }^{2}$ Department of Physics, West Virginia University, Morgantown, West Virginia 26506, USA

${ }^{3}$ Institute of Electrical Engineering and Plasma Technology, Ruhr-University Bochum, 44780 Bochum, Germany

${ }^{4}$ Institute for Solid State Physics and Optics, Wigner Research Centre for Physics, Hungarian Academy of Sciences, 1121 Budapest, Hungary

${ }^{5}$ Department of Physics, Chungnam National University, Daejeon 34134, South Korea
\end{abstract}

a) LHC@kriss.re.kr

\begin{abstract}
Geometrically symmetric capacitively coupled oxygen plasmas are studied experimentally by optical emission spectroscopy and probe measurements as well as via numerical simulations using the kinetic Particle-in-Cell/Monte Carlo collision (PIC/MCC) approach. The experiments reveal that at a fixed pressure of 20 mTorr and a driving frequency of $13.56 \mathrm{MHz}$, the central electron density increases with an increased electrode gap, while the time averaged optical emission of atomic oxygen lines decreases. These results are reproduced and understood by the PIC/MCC simulations performed under identical conditions. The simulations show that the electron density increases due to a mode transition from the Drift-Ambipolar-mode to the $\alpha$-mode induced by increasing the electrode gap. This mode transition is due to a drastic change of the electronegativity and the mean electron energy, which leads to the observed reduction of the emission intensity of an atomic oxygen line. The observed mode transition is also found to cause a complex non-monotonic dependence of the $\mathrm{O}_{2}^{+}$ion flux to the electrodes as a function of the electrode gap. These fundamental results are correlated with measurements of the etch rate of amorphous carbon layers at different gap distances.
\end{abstract}

Published under license by AIP Publishing. https://doi.org/10.1063/1.5063543

\section{INTRODUCTION}

Low temperature radio frequency $(\mathrm{RF})$ plasmas operated in gas mixtures containing oxygen at low pressures of a few $\mathrm{Pa}$ or below have widely been used in industrial semiconductor and display processing for etching, ashing, and deposition. ${ }^{1-7}$ In order to improve the device performance and the production yield, feature sizes must be shrunk and new device structures with three-dimensional (3D) architectures must be developed. Important applications are the production of Fin Field-effect transistors (Fin-FETs), 3D vertical NAND flash memory, and 3D integrated dynamic RAM (DRAM). ${ }^{5}$ To fabricate these complicated structures, advanced plasmaetching techniques, e.g., high aspect ratio and damage free etching, are required.
The basis of this wide variety of applications is the synergistic effect of ions and radicals on boundary surfaces. These species originate from various plasma-chemical processes at low heavy-particle temperatures. ${ }^{4}$ For further process optimization, a deep fundamental understanding of the plasma processes and characteristics is required. This includes the dependence of important plasma parameters such as the electron density, mean electron energy, electronegativity, and flux-energy distribution functions of different particle species on external control parameters such as the RF power/voltage, the driving frequency, the gas pressure, and the discharge gap. Only such a fundamental understanding allows the development of precise control techniques in current and next generation plasma processing. Such parameter trends are not understood and 
sometimes not even known for relatively simple gases/gas mixtures since the corresponding plasma characteristics are not measured and/or studied computationally. In addition to their industrial relevance, such insights also play a major role in fundamental low temperature plasma science.

In order to realize such a "knowledge-based" process optimization, access to a variety of discharge characteristics is required. As typically only a fraction of these plasma parameters can be measured, modeling studies and/or computational simulations are important. At low pressures, kinetic models/simulations are required. However, it is necessary to benchmark these tools against experiments to make sure that they describe the discharge conditions of interest realistically. Finally, trends of process relevant plasma parameters must be correlated with the characteristics of deposited/etched films on the wafer.

Such studies are required for a variety of plasma sources and discharge conditions, which represent a major challenge for future research, since currently only very few plasma sources and discharge conditions are understood. For each plasma source and given discharge conditions, insights into the electron power absorption dynamics as a function of external control parameters and their consequences on plasma parameters as well as film characteristics have to be obtained.

In capacitively coupled plasmas (CCPs), some knowledgebased studies on the effects of external control parameters on plasma characteristics have been performed in the past. For instance, the effects of the driving frequency on the plasma density were studied for selected discharge conditions. ${ }^{11-15}$ While in a wide range of conditions, an increase in the plasma density as a function of the square of the driving frequency was found due to enhanced electron heating, Wilczek et al. ${ }^{15}$ observed a step-like increase in the plasma density above a threshold driving frequency at low neutral gas pressure in the non-local regime. ${ }^{16}$ This step-like increase was found to occur because electron beams generated by sheath expansion at one electrode impinge at the opposite electrode during the local sheath collapse for distinct driving frequencies, and, therefore, a large number of energetic electrons are lost and the plasma density is low. For other driving frequencies, such beam electrons impinge at the opposite electrode at a different time within the RF period, when the local sheath is expanded, and are, therefore, reflected and confined well, resulting in an enhanced electron density. Perret et al. ${ }^{14}$ observed strong lateral non-uniformities of the ion flux across large electrodes in CCPs operated at high driving frequencies due to electromagnetic effects such as the standing wave and the skin effect. ${ }^{17}$

There are various modes of discharge operation of CCPs ranging from the $\alpha^{-18-20}$ and $\gamma^{-19,20}$ to the Drift-Ambipolar (DA)- ${ }^{21}$ and the Striation-modes. ${ }^{22-24}$ The effects of changing some external control parameters on transitions between these modes were clarified for selected discharge conditions. Multifrequency CCPs operated with tailored voltage waveforms were found to provide additional and separate control of various plasma characteristics such as the electron heating dynamics, the DC self-bias, and particle flux-energy distribution functions of different particle species at boundary surfaces and in the plasma volume by customizing the driving voltage waveform. ${ }^{25-31}$ Similar studies were performed in other plasma sources such as inductively coupled plasmas with capacitive RF substrate bias, where important coupling mechanisms between the inductive and capacitive power on the spatio-temporal electron heating dynamics, the plasma density, and ion-flux energy distribution functions at the wafer were found. ${ }^{32-42}$ In CCPs operated in $\mathrm{O}_{2}$, Dittmann and Matyash et al. ${ }^{43,44}$ as well as Kuellig et al. ${ }^{45}$ studied the effects of changing the driving voltage amplitude and the neutral gas pressure on the electron power absorption dynamics as well as instabilities in single-frequency discharges.

Concerning applications, Sung et al. ${ }^{46}$ observed an etch rate nonuniformity as a function of the driving frequency and power as a consequence of electromagnetic effects in CCPs. Johnson et al. ${ }^{47,48}$ developed a method to change the growth property of thin silicon films from amorphous to nanocrystalline via voltage waveform tailoring. Neyts et al. ${ }^{49}$ observed a defect healing and enhanced cap formation on carbon nanotubes induced by low ion energy bombardment. Lee et al. ${ }^{37}$ found that applying a low inductive power in hybrid inductively and capacitively coupled plasmas allows us to realize an independent control of the plasma density and electron temperature as well as obtaining an improved photoresist ashing with minimum damage of sensitive materials.

Systematic studies of the effects of the electrode gap on plasma parameters in CCPs are limited, although this external control parameter plays a key role in the design of plasma sources. Jiang et al. $^{50}$ investigated the effects of the gap length in argon discharges via Particle-in-Cell/Monte-Carlo Collisions (PIC/MCC) simulations and showed that a hysteresis of the plasma density as a function of the electrode gap exists. You et $a .^{51}$ studied the dependence of the electron energy distribution function (EEDF) in argon plasmas on the electrode gap length and found changes in the EEDF from bi-Maxwellian to Maxwellian induced by changing the gap due to heating of the low energy electrons by the enhanced bulk electric fields.

Investigations of the effects of changing the electrode gap on discharge characteristics and film properties are particularly limited in CCPs operated in oxygen, although such plasma sources are often used for applications, e.g., the etching of highly oriented pyrolytic graphite ${ }^{52}$ and polydimethyl siloxane films, ${ }^{53}$ for resist stripping and multilayer lithography, ${ }^{54}$ and for etching of graphene ${ }^{55}$ and polycyclohexene carbonate. ${ }^{56}$ As mentioned above, in order to gain access to various discharge characteristics such experimental studies should also be accompanied by modeling/simulations, which is now possible with accurate kinetic PIC/MCC codes developed recently. ${ }^{44,57-61}$

Therefore, in this work, we investigate the effects of changing the electrode gap on important plasma characteristics such as the central electron density, electronegativity, mean electron energy, and ion flux-energy distribution functions in low pressure single-frequency CCPs operated at $13.56 \mathrm{MHz}$ in $\mathrm{O}_{2}$. We also study the time averaged optical emission from atomic oxygen lines and the spatio-temporal ionization dynamics. These investigations are performed based on a synergistic combination of experiments and computational (PIC/MCC) simulations. In 
this way, a detailed fundamental understanding of the plasma physics is gained and an experimental validation of the simulations is performed. These fundamental results are correlated with measurements of the etch rate of amorphous carbon layers (ACLs).

This paper is structured in the following way: In Sec. II, we describe the experimental setup including all diagnostics. In Sec. III, the computational model is introduced. Our results are presented in Sec. IV, which is split into two parts. In the first part (Sec. IVA), we present our experimental and computation results with regard to the effects of changing the electrode gap on plasma parameters. In the second part (Sec. IV B), these fundamental findings are correlated with the measured ACL etch rates. Finally, conclusions are drawn in Sec.V.

\section{EXPERIMENTAL SETUP}

Figure 1 shows a schematic diagram of the experimental setup. The reactor has a cylindrical shape with an inner diameter of $26 \mathrm{~cm}$ and a height of $30 \mathrm{~cm}$ and is made of quartz. The two electrodes, having a diameter of $12 \mathrm{~cm}$, are made of stainless steel, and their surfaces are coated with aluminium oxide. To prevent coupling of the RF power between the electrodes and the sidewall, the electrodes have been covered with a thick ceramic insulator material except their surfaces facing each other. The RF power at a frequency of $13.56 \mathrm{MHz}$ is applied to the upper electrode through an L-type impedance matching

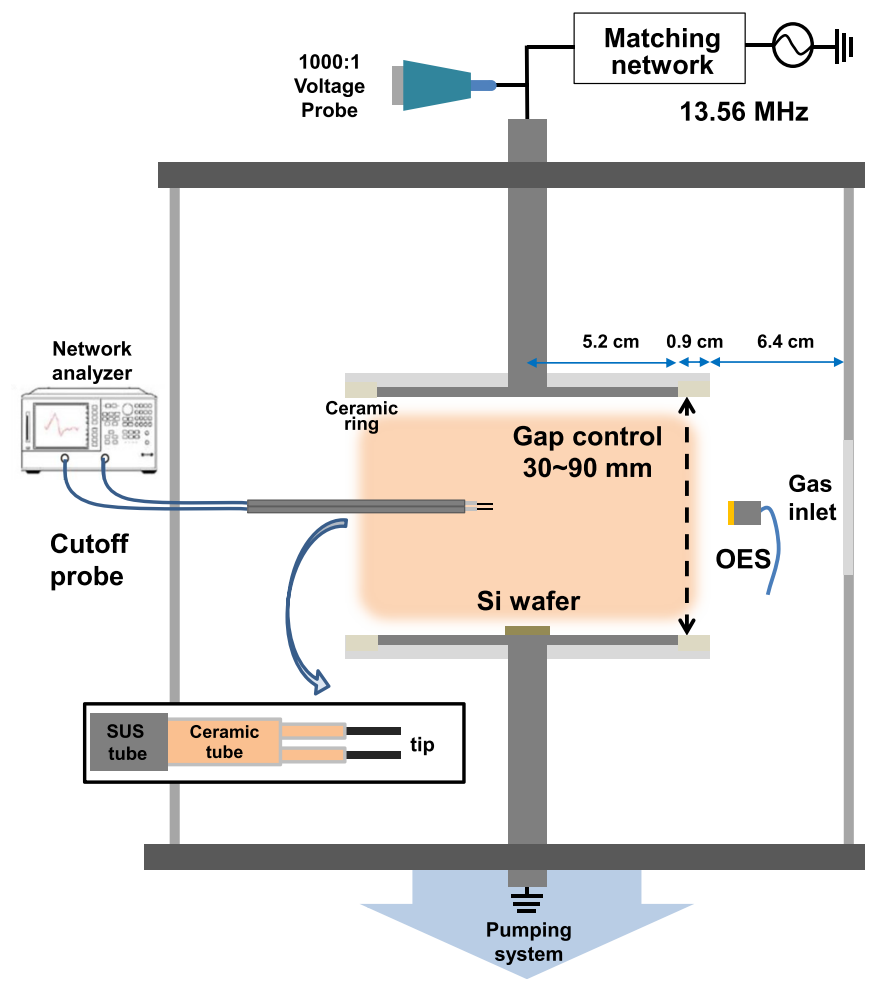

FIG. 1. Experimental setup of the RF CCP reactor, including the diagnostics system. The etching studies are performed on amorphous carbon layers deposited on Si wafers, prior to the experiments. network, while the lower electrode is grounded. A high voltage probe (P6015A, Tektronix Inc.) is mounted at the powered electrode to measure the voltage drop across the discharge. Due to the symmetry of the system, no DC self-bias voltage is expected to be generated. Nevertheless, during the measurements, its value is monitored. Such a geometrically symmetric reactor is used to allow comparisons of the experimental results with $1 \mathrm{~d} 3 \mathrm{v}$ PIC/MCC simulations of such a parallel plate system, which inherently assumes an ideal geometric reactor symmetry.

The base pressure in the reactor is less than $7 \times 10^{-4}$ Torr. The $\mathrm{O}_{2}$ gas is injected into the plasma chamber through a mass flow controller, and its pressure is monitored using a capacitor diaphragm gauge (MKS Inc.) mounted at the chamber wall. The experiments are conducted at a pressure of 20 mTorr and at a gas flow rate of $11 \mathrm{sccm}$. The positions of the upper and lower electrodes can be adjusted in the vertical direction using a motorised system, allowing electrode separations between $\mathrm{L}=0 \mathrm{~mm}$ and $90 \mathrm{~mm}$. In the present study, the plasma operating conditions limit the gap size to $30 \mathrm{~mm} \leq$ $\mathrm{L} \leq 90 \mathrm{~mm}$. The driving voltage amplitude is kept constant at $300 \mathrm{~V}$.

The electron density is measured using a microwave cutoff probe placed at the center of the chamber at the midpoint between the electrodes. This probe consists of two coaxial cables in a stainless steel holder. One coaxial cable is a radiating antenna, while the other is a detection antenna. Each tip is $5 \mathrm{~mm}$ long and has a diameter of $0.1 \mathrm{~mm}$, and both tips are located $\approx$ $2 \mathrm{~mm}$ apart from each other. This probe allows absolute electron density measurements, while due to its small size, the plasma density depletion caused by its presence in the plasma is low. The cutoff probe is connected to a network analyzer. To measure the plasma density, one antenna radiates a frequencyswept signal at a power of $1 \mathrm{~mW}$ and this signal is captured by the other antenna above a certain threshold frequency corresponding to the electron plasma frequency (due to the wave cutoff in the plasma medium). Thus, from the measured signal, the electron plasma frequency, $\omega_{\text {pe }}$, can be obtained and the electron density, $n_{\mathrm{e}}$, can be determined using the following relationship: $:^{62,63}$

$$
\omega_{\text {cutoff }}=\omega_{\text {pe }}=\left(\frac{n_{\mathrm{e}} e^{2}}{\epsilon_{0} m}\right)^{1 / 2} .
$$

Here, $m$ and $e$ are the electron mass and the elementary charge, respectively. The time averaged optical emission spectrum of the plasma is measured at a view port using an Ocean Optics Inc. HR 4000 spectrometer. Light is collected from a radially centered position, which is adjustable in the vertical direction using an optical stage, to allow scanning the light emission intensity within the electrode gap.

In order to investigate the etch rate of ACL, samples of ACLs are prepared by plasma enhanced chemical vapor deposition (PECVD) on silicon wafers with a size of $2 \mathrm{~cm} \times 2 \mathrm{~cm}$. The deposition is performed in a commercial PECVD reactor $(\mathrm{P} 5000$, AMAT Inc.) at a pressure of 7Torr using a gas mixture of $\mathrm{C}_{2} \mathrm{H}_{2}$ (250 sccm), Ar (1700 sccm), He (500 sccm), and $\mathrm{N}_{2}(700 \mathrm{sccm})$. The temperature of the PECVD chamber is kept at $400^{\circ} \mathrm{C}$, and the RF power is set to $700 \mathrm{~W}$ at $13.56 \mathrm{MHz}$. The initial thickness 
of the deposited ACL hard mask on the silicon wafer is $2 \mu \mathrm{m}$ as verified by scanning electron microscopy (SEM). For the etching experiments, these wafers are placed onto the lower electrode to study the etch rate. A different wafer is used for each value of the electrode gap. The wafers are exposed to the $\mathrm{O}_{2}$ plasma for $10 \mathrm{~min}$, and subsequently, the etch rate is deduced from SEM analysis of the wafers following the plasma treatment.

\section{SIMULATION METHOD}

Complementing the experimental studies, we also conduct computational investigations of the oxygen discharges for identical conditions as in the experiments. In this way, the simulation results are benchmarked experimentally and access to a variety of plasma parameters is obtained, which are crucially important for a detailed understanding of the plasma physics, but cannot be measured. These numerical studies are based on our Particle-in-Cell/Monte Carlo Collision code developed earlier for low-pressure capacitive RF oxygen discharges. ${ }^{57}$ The code traces electrons, $\mathrm{O}_{2}^{+}$ions, and $\mathrm{O}^{-}$ions in a homogeneous background of oxygen gas. The motion of the charged particles is traced in a 3D velocity space, while a single spatial coordinate is considered only. (This is certainly a limitation of the code that may result in a less accurate comparison between the experimental and simulation results when the gap length in the experiment becomes comparable to the electrode diameter.)

The set of elementary collision processes follows to a large extent the "xpdp1" set. ${ }^{64}$ Compared to the original xpdp1 set, however, we replace the elastic collision cross-section with the elastic momentum transfer cross-section of Biagi ${ }^{65}$ and use, accordingly, isotropic electron scattering. Moreover, we substitute the original xpdp1 ionisation cross-section with that recommended by Gudmundsson et al. ${ }^{66}$ and adopt the cross-sections for all the heavy particle (ion-molecule and ion-ion collision) processes from the same work, ${ }^{66}$ except the cross-section of the direct detachment process $\mathrm{O}^{-}+\mathrm{O}_{2} \rightarrow \mathrm{O}+\mathrm{O}_{2}+\mathrm{e}^{-}$that is taken from the work of Bronold et al. ${ }^{67}$ An estimation of the density of the singlet delta $\mathrm{O}_{2}$ excited molecules is also necessary in the model because via the $\mathrm{O}^{-}+\mathrm{O}_{2}\left(\mathrm{a}^{1} \Delta_{\mathrm{g}}\right) \rightarrow \mathrm{O}_{3}+\mathrm{e}^{-}$associative detachment process, molecules in this excited state can influence the $\mathrm{O}^{-}$ion density and this way the electronegativity of the plasma. We perform this estimation by balancing the source of molecules in this excited state and their losses due to surface recombination. For the surface recombination rate, we use $\alpha=6 \times 10^{-3}$ (Refs. 57,61, and 68). (We note that data for this coefficient show large scattering in the literature. ${ }^{58,67,69,70}$ For a recent study of the effect of metastable molecules in oxygen plasmas, see Ref. 71.) The ion induced secondary electron emission coefficient is set to $\gamma=0.4$, which is expected to characterize well the electron emitting properties of the oxidized electrode surfaces. While we do not consider secondary electron emission from the electrodes by electron impact, ${ }^{72,73}$ electrons can be elastically reflected from these surfaces with a constant probability of $\rho=0.2 .^{74}$ The gas temperature is fixed at $\mathrm{T}_{\mathrm{g}}=300 \mathrm{~K}$.

For more details about the reaction set and the computational implementation, see Refs. 57 and 61, which also report benchmarking of the present code with an extensive set of experimental data. A limited sensitivity analysis regarding some of the input data has also been reported based on this code. ${ }^{68}$

The computations are conducted by paying attention to the relevant stability criteria of the PIC/MCC method. The numerical grid has divisions between $M=500$ (short gaps) and 2500 (long gaps), and the time step is chosen to be $1 / 5000$ part of the $\mathrm{RF}$ period $\mathrm{T}_{\mathrm{RF}}=1 / f$. These parameter settings ensure that the spatial grid resolves the Debye length and that the time step resolves the electron plasma oscillations, for all the conditions studied. The simulations allow the determination of a large set of discharge characteristics. The results will only be shown for the charged particle densities, electronegativity, mean electron energy, the flux-energy distribution function of $\mathrm{O}_{2}^{+}$ions at the electrodes, the spatio-temporal ionization and dissociative attachment rates, and the time averaged excitation rate of the upper level of the $\lambda=844.6 \mathrm{~nm}$ atomic oxygen transition that results from the dissociative excitation of $\mathrm{O}_{2}$ molecules via the $\mathrm{e}^{-}+\mathrm{O}_{2} \rightarrow \mathrm{e}^{-}+\mathrm{O}+\mathrm{O}\left(3 p^{3} \mathrm{P}\right)$ process. We note that optical emission resulting from this transition may also originate by electron impact excitation of atomic oxygen (created by different dissociative processes), but as our model does not include the balance equation for the $\mathrm{O}$ atom density, we cannot account for this part of the excitation.

\section{RESULTS}

First, we investigate the effect of changing the electrode gap on the plasma fundamentally, based on measurements and PIC/MCC simulations performed under identical conditions. These results are presented in Sec. IVA and yield a detailed fundamental understanding of CCPs operated in $\mathrm{O}_{2}$. Based on these insights, in Sec. IV B, we study the effects of changing the electrode gap on the ACL etch rate experimentally and correlate these process relevant findings with the fundamental understanding of the plasma physics obtained initially. As mentioned earlier, the discharge is driven at $13.56 \mathrm{MHz}$ with a constant driving voltage amplitude of $300 \mathrm{~V}$, the neutral gas pressure is 20 mTorr, and the electrode gap is varied from $30 \mathrm{~mm}$ to $90 \mathrm{~mm}$.

\section{A. The effect of changing the electrode gap on plasma parameters}

Figure 2(a) shows the measured and computed electron densities in the center of the discharge as a function of the electrode gap. Good quantitative agreement between the experimental and simulation results is obtained except for the smallest electrode gaps. These differences can be explained by the limited accuracy of the simulation due to, e.g., the uncertainty of the choice of the surface coefficients as input parameters (e.g., secondary electron emission, particle reflection, and loss probabilities at boundary surfaces). The electron density is found to depend on the electrode gap in a complex non-linear way, i.e., we observe a weak increase at small gaps followed by a strong increase around $L \sim 50 \mathrm{~mm}$, which is again followed by a weaker increase at larger gap distances.

Figure 2(b) shows the measured time averaged optical emission intensity of the atomic oxygen line at $844.6 \mathrm{~nm}$ in the discharge center as a function of the electrode gap. It also shows 

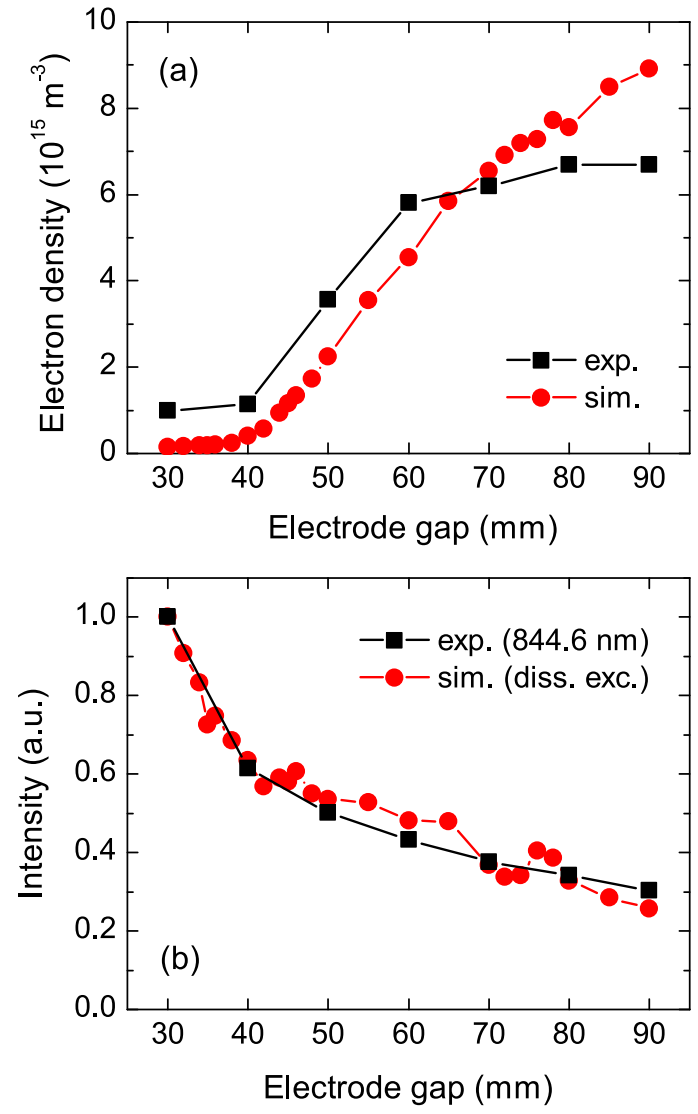

FIG. 2. (a) Measured and computed electron densities in the discharge center and (b) measured optical emission intensity at $844.6 \mathrm{~nm}$ at the center of the bulk plasma and the rate of dissociative excitation of the upper state of the $844.6 \mathrm{~nm}$ oxygen line obtained from PIC/MCC simulations as a function of the electrode gap.

the computed electron impact excitation rate from the molecular ground state into the upper state of the $844.6 \mathrm{~nm}$ atomic oxygen transition resulting from dissociative excitation of oxygen molecules. This rate is computed within a $5 \mathrm{~mm}$ wide region centered around the mid-plane of the discharge, i.e., it characterizes the excitation rate in the bulk plasma as a function of the gap length. Both quantities are provided in arbitrary units. Excellent agreement between the experimental and the simulation results is found. The measured optical emission and the computed dissociative electron impact excitation rate decrease as a function of the electrode gap. In the presence of an increasing electron density, this result predicts a decrease in the atomic oxygen density as a function of the gap distance.

In order to understand these findings, access to additional plasma parameters is required-these are provided by the simulation. Figure 3(a) shows the mean electron energy and the electronegativity in the center of the discharge as a function of the electrode gap. Here, the electronegativity is defined as the ratio of the local negative ion and electron densities. The mean electron energy decreases from about $10 \mathrm{eV}$ at small electrode gaps to about $1 \mathrm{eV}$ at the largest electrode gap of $90 \mathrm{~mm}$. In parallel,
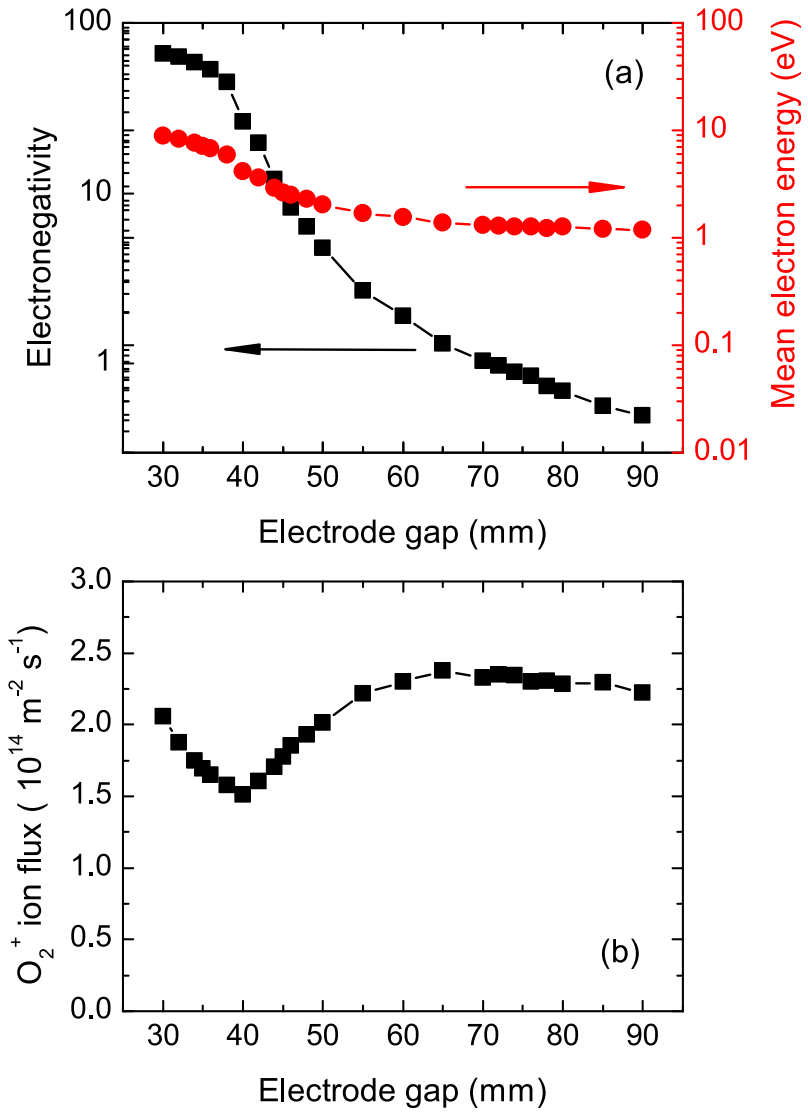

FIG. 3. PIC/MCC simulation results: (a) electronegativity of the discharge (left vertical scale) and the mean electron energy in the discharge center (right vertical scale) and (b) flux of the $\mathrm{O}_{2}^{+}$ions at the electrodes as a function of the gap length.

the $\mathrm{O}_{2}^{+}$ion flux to the electrodes initially decreases and then increases as a function of the electrode gap [see Fig. 3(b)]. This behaviour will be discussed below.

Figure 4 shows the time averaged charged particle density profiles of electrons, $\mathrm{O}_{2}^{+}$ions, and $\mathrm{O}^{-}$ions at different electrode gaps of $30 \mathrm{~mm}, 40 \mathrm{~mm}, 50 \mathrm{~mm}$, and $80 \mathrm{~mm}$ as well as the densities of these species in the discharge center as a function of the electrode gap. Figure $4(\mathrm{e})$ shows that the electronegativity in the discharge center decreases as a function of the gap length, since the negative ion density decreases and the electron density increases, while the positive ion density increases only slightly. These trends are caused by the following mechanism: at a small electrode gap of $30 \mathrm{~mm}$, the discharge is strongly electronegative and the charged particle density profiles have shapes typical for electronegative CCPs, i.e., the electron density has local maxima in the electropositive edge regions of the plasma. ${ }^{1}$ This leads to an operation of the discharge in the DA-mode because electrons are accelerated by strong drift electric fields in the bulk at the times of maximum RF current due to the depleted conductivity and by strong ambipolar fields caused by the presence of electron density maxima close to the position of the maximum sheath width. ${ }^{21}$ The presence of the DA-mode under 

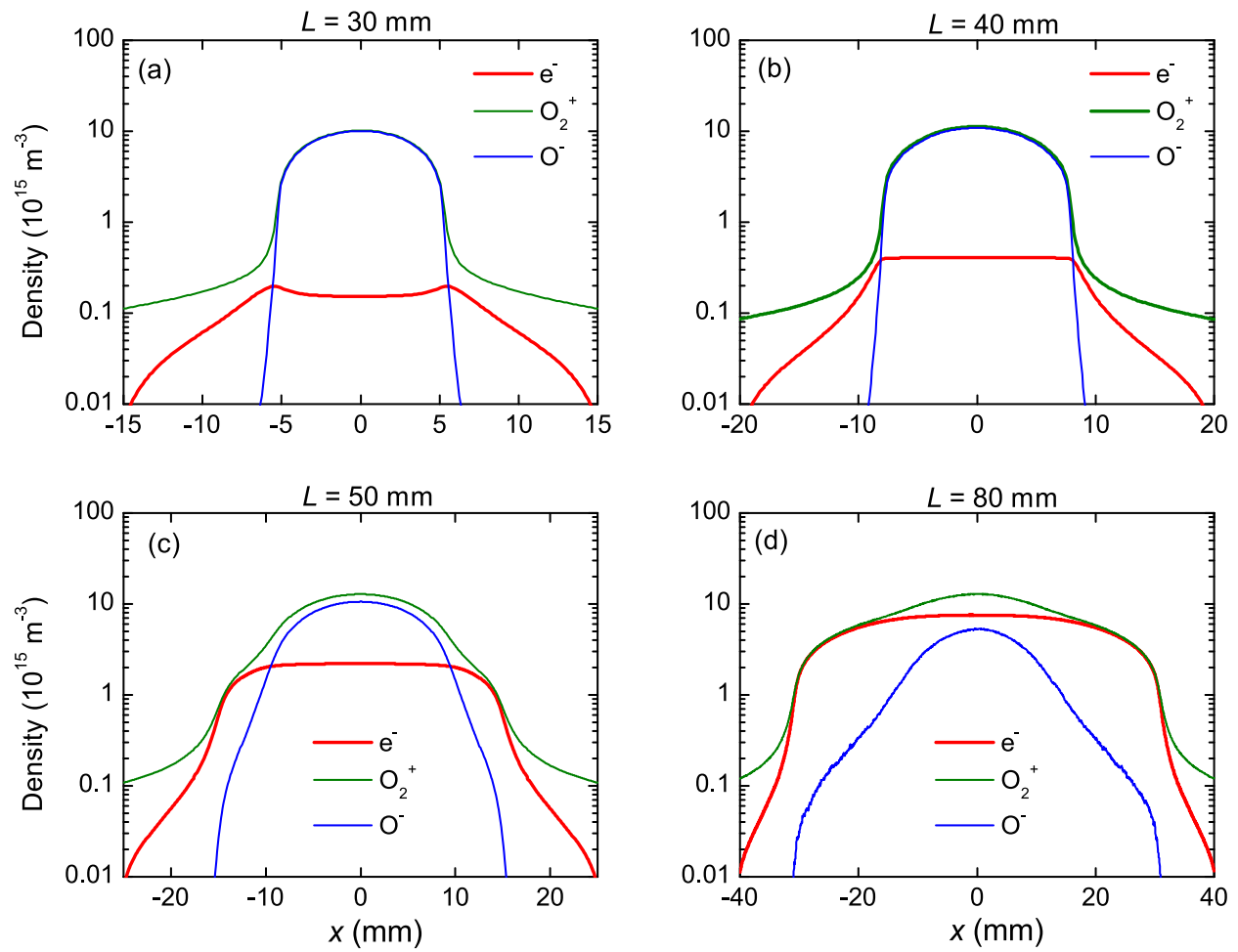

FIG. 4. PIC/MCC simulation results: (a)-(d) time averaged charged particle density distributions for different gap lengths and (e) the density of the charged particles in the discharge center as a function of the gap length.

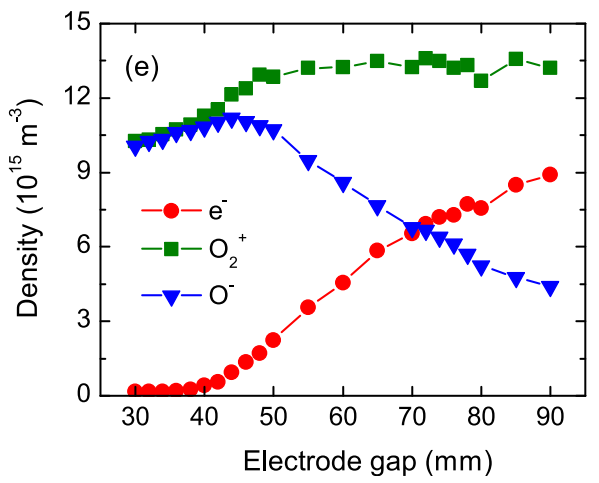

these discharge conditions is clearly observed in Fig. 5(a), which shows strong ionization maxima on the bulk side of the collapsing sheaths caused by the acceleration of electrons towards the electrodes primarily by ambipolar electric fields. Thus, electrons are accelerated to high energies in regions close to the maximum sheath width. This, in turn, leads to a high rate of generation of negative ions via electron attachment in these regions [see Fig. 6(a)] because the cross-section for dissociative electron attachment peaks at relatively high electron energies of 6-7 eV. Thus, the negative ion sources are located inside the sheath for some fraction of the RF period, when the local sheath is expanded, and outside the sheath during the rest of the RF period. As the negative ions created in these regions can only react to the time averaged local electric field, they are accelerated towards the discharge center. This happens at both electrodes. As the sheaths cover a large fraction of the discharge volume at short electrode gaps, the negative ions are confined to a relatively small plasma bulk volume and the negative ion density is high in the discharge center. Due to quasineutrality in the bulk, the electron density is low for a given positive ion density.

Increasing the electrode gap causes the volume of the quasineutral plasma bulk to cover a larger fraction of the total discharge volume, which results in a decrease in the negative ion density in the bulk. This can be understood in the following way: for small gap distances, the negative ion sources are located at the positions of the maximum sheath edges. After increasing the gap distance abruptly, these negative ion sources are initially the same in the simulation, but a larger bulk volume is filled by negative ions predominantly generated by these sources. Thus, the electronegativity in the bulk decreases.

For a given positive ion density, this leads to an increase in the electron density as a function of the electrode gap. This, in 

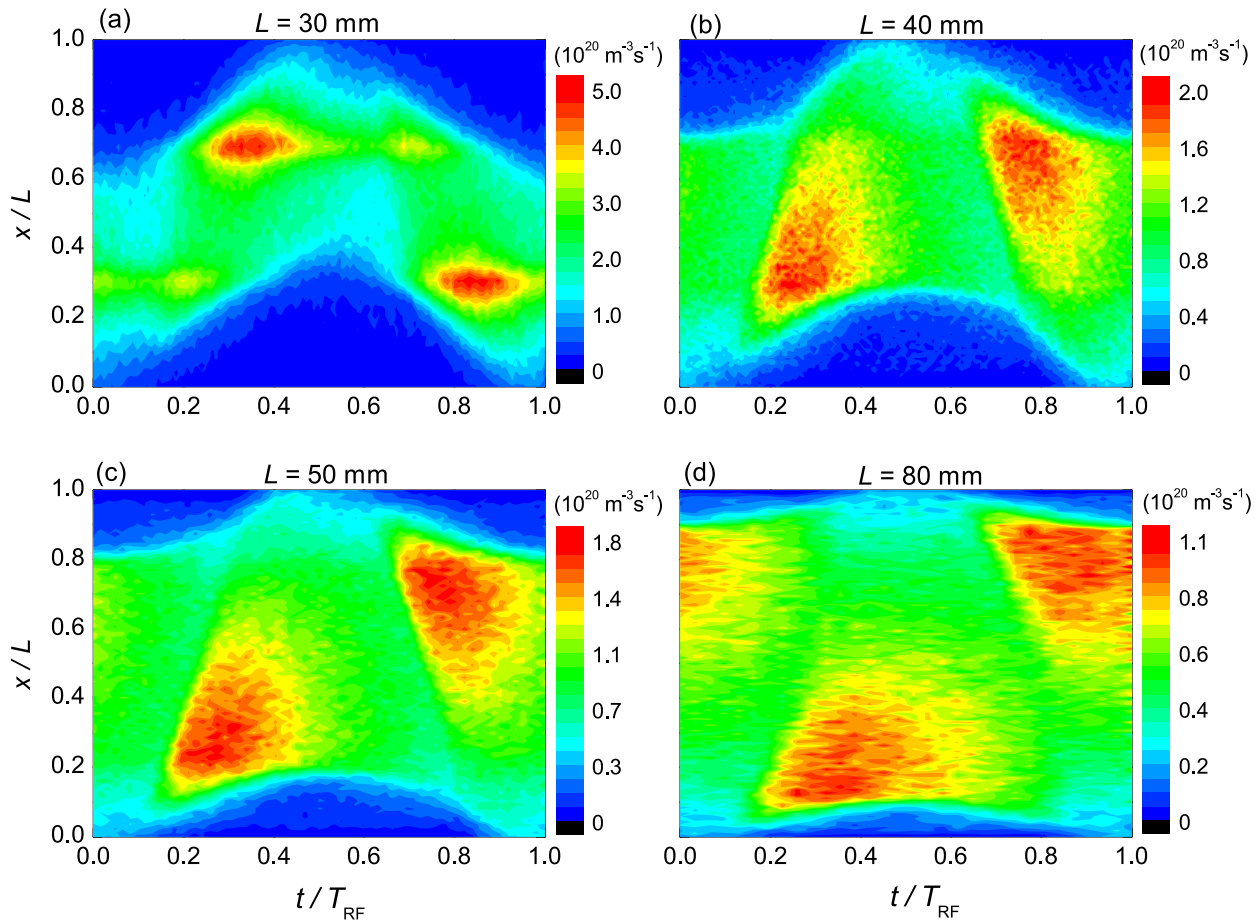

FIG. 5. PIC/MCC simulation results: spatio-temporal distribution of the ionization rate for different gap lengths.
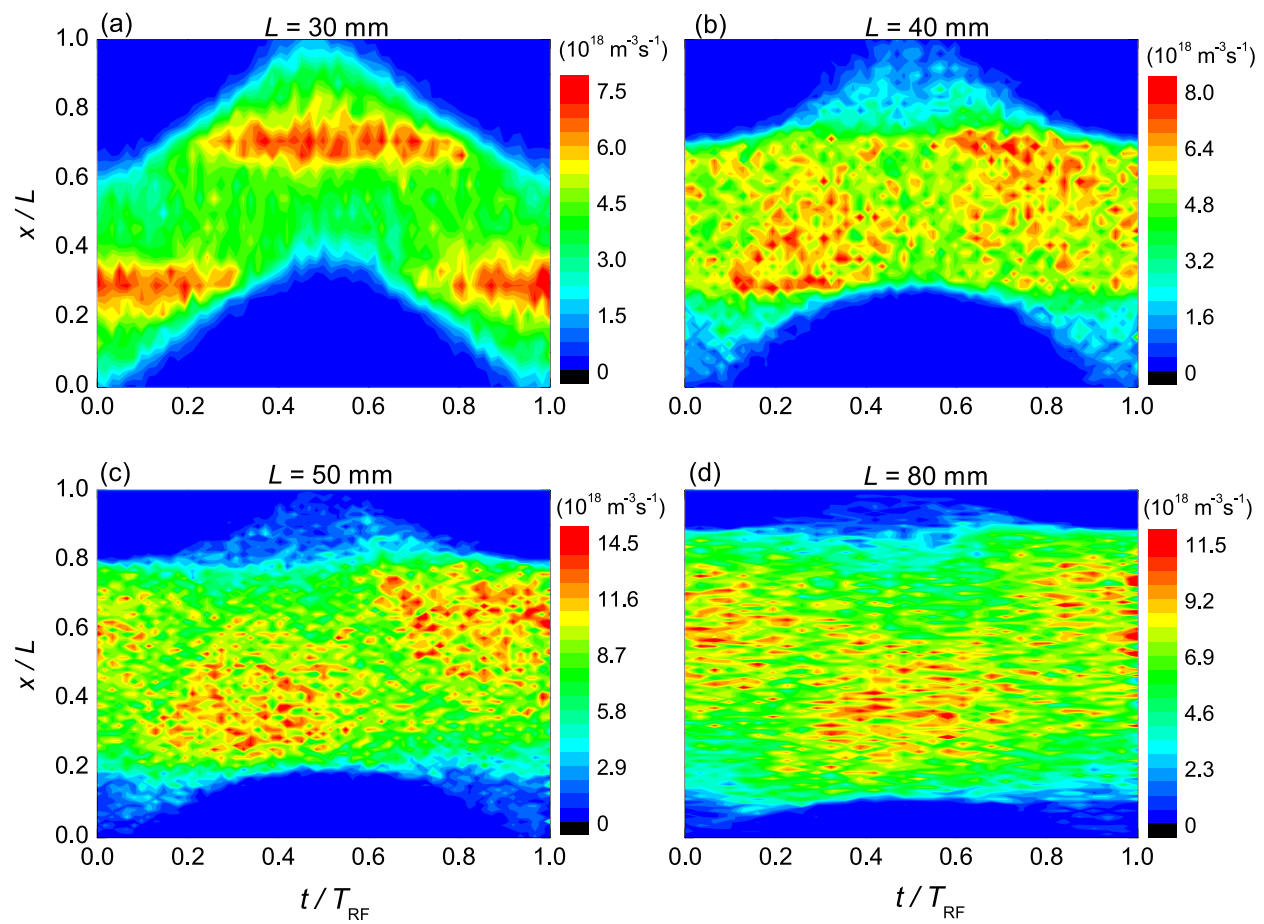

FIG. 6. PIC/MCC simulation results: spatio-temporal distribution of the dissociative attachment rate for different gap lengths.

turn, results in lower drift and ambipolar electric fields because the conductivity is enhanced and the local extrema of the electron density close to the positions of the maximum sheath width vanish [see Figs. 4(b)-4(d)]. This induces a mode transition from the DA-mode to the $\alpha$-mode, where sheath expansion heating of electrons dominates [see Figs. 5(b)-5(d)]. It also leads to a vanishing of the strong negative ion sources at the positions of maximum sheath widths at both electrodes [see Figs. 6(b)-6(d)]. 
Instead, negative ions are predominantly formed inside the plasma bulk for large gap distances, because in the $\alpha$-mode, energetic beam electrons generated by sheath expansion heating propagate into the plasma bulk and the cross-section for negative ion formation via dissociative attachment peaks at relatively high electron energy. Thus, the negative ion formation is determined by the corresponding part of the electron energy distribution function, which changes due to the mode transition from the DA- to the $\alpha$-mode induced by changing the electrode gap. Overall, this is a self-amplifying mechanism that causes the observed changes of the electron power absorption dynamics as well as the observed changes of the electronegativity and the charged particle densities in the discharge center as a function of the electrode gap.

The $\mathrm{O}_{2}^{+}$ion flux at the electrodes is found to decrease initially and then to increase as a function of the electrode gap in Fig. 3(b), while a slight monotonous increase in the positive ion density in the discharge center is observed in Fig. 4(e). The initial decrease in the positive ion flux is also caused by the mode transition of the electron power absorption induced by changing the electrode gap. At a short gap distance, the discharge is operated in the DA-mode and the ionization rate peaks close to the electrodes on the bulk side of the collapsing sheath edge [see Fig. 5(a)]. Thus, positive ions are generated close to the electrodes and are immediately accelerated towards the adjacent boundary surface by the time averaged local electric field. Thus, the ion flux to the electrodes is high, although the central positive ion density is low. Increasing the electrode gap induces a mode transition into the $\alpha$-mode [see Figs. $5(\mathrm{~b})-5(\mathrm{~d})$ ]. Thus, positive ions are generated further inside the bulk in regions where they are no longer accelerated to the electrodes by the time averaged sheath electric field. Thus, the ion flux to the electrodes initially decreases as a function of the electrode gap and then follows the trend of the central positive ion density, i.e., it increases as a function of the gap distance at large electrode gaps.

Figure 7 shows the $\mathrm{O}_{2}^{+}$ion flux-energy distribution functions (IFEDF) at the electrodes for different electrode gaps, which is relevant for plasma surface interactions. At the low operating pressure of 20 mTorr, the sheaths are almost

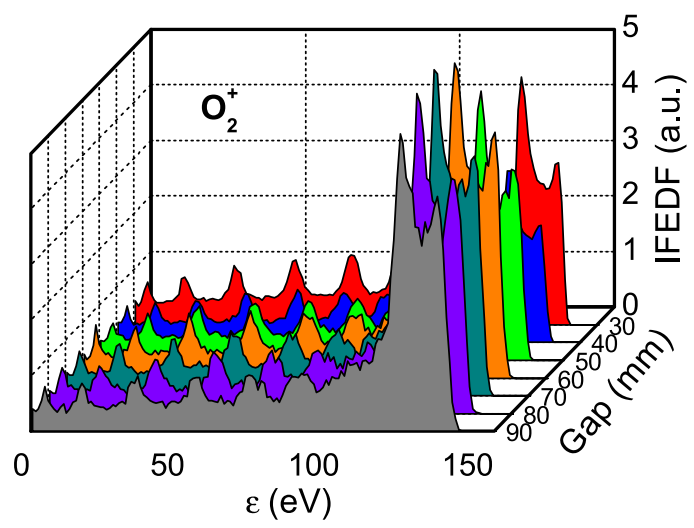

FIG. 7. PIC/MCC simulation results: IFEDF of $\mathrm{O}_{2}^{+}$ions at the electrodes for different gap lengths. collisionless so that a dominant high energy bi-modal peak at around $130 \mathrm{eV}$ is formed-this shape indicates that the $\mathrm{O}_{2}^{+}$ions can react to the instantaneous sheath electric field to a limited extent. At lower energies we observe a sequence of small amplitude peaks, which are caused by charge-exchange collisions of the $\mathrm{O}_{2}^{+}$ions inside the sheath.

\section{B. The effect of changing the electrode gap on amorphous carbon layer etching}

The fundamental results obtained in Sec. IVA indicate significant changes in important plasma parameters as a function of the electrode gap, which are expected to affect the plasma processing performance. Here, we study the effect of changing the electrode gap on the ACL etch rate experimentally. Samples of ACLs with a thickness of $2 \mu \mathrm{m}$ are deposited on silicon wafers according to the procedure described in Sec. II. These wafers are then placed onto the lower electrode and are exposed to an $\mathrm{O}_{2}$ plasma for 10 min under the conditions studied in the Sec. IVA using different electrode gaps. For each gap, a new wafer is used. Subsequently, the etch rate is measured by SEM analysis of the wafers following the plasma treatment. SEM images of a reference ACL-covered wafer and the etched wafers are shown in Fig. 8(a), while the measured film thickness and the deduced etch rate are displayed in Fig. 8(b) as a function of the gap length.

(a)
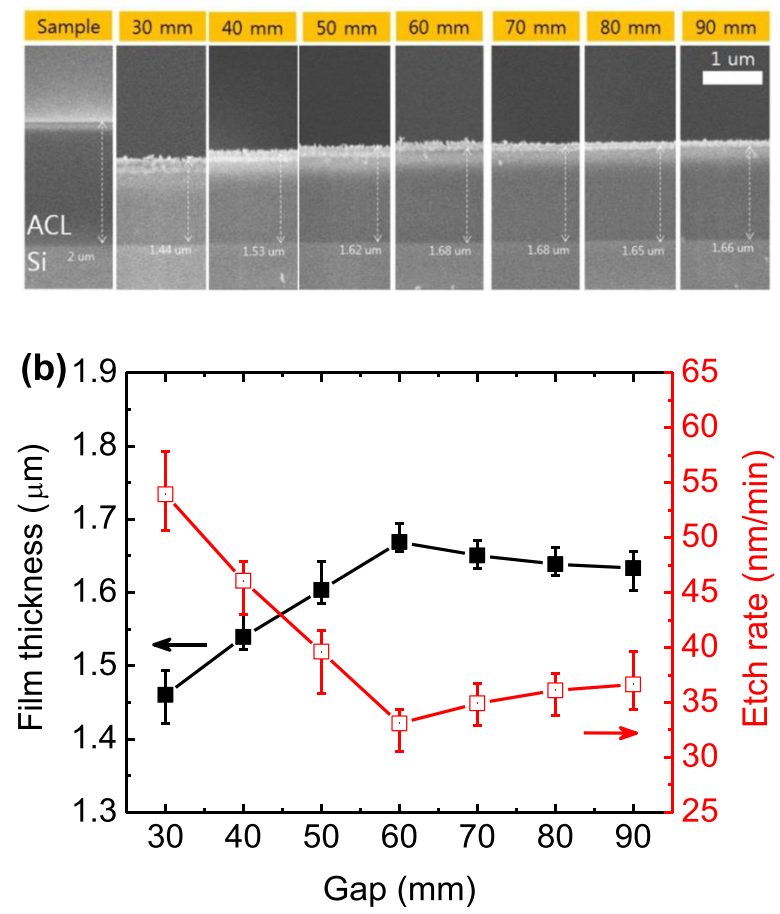

FIG. 8. (a) SEM images of the ACL hard mask etching and (b) film thickness as well as the ACL etch rate for different electrode gaps. Discharge conditions: CCP operated in $\mathrm{O}_{2}$ at $13.56 \mathrm{MHz}, 20 \mathrm{mTorr}, 300 \mathrm{~V}$ driving voltage amplitude. 
The film thickness at the electrode gap of $30 \mathrm{~mm}$ is about $1.46 \mu \mathrm{m}$, which implies that approximately $0.54 \mu \mathrm{m}$ of the ACL was etched during the plasma exposure of $10 \mathrm{~min}$. As the gap distance is increased from $30 \mathrm{~mm}$ to $60 \mathrm{~mm}$, the film thickness increases monotonically, implying a reduction in the etch rate. As the gap length increases from $60 \mathrm{~mm}$ to $90 \mathrm{~mm}$, a slight increase in the etch rate is found. Thus, a minimum of the etch rate is found at an electrode gap of $60 \mathrm{~mm}$.

While changing the electrode gap, the DC self-bias remains at approximately $0 \mathrm{~V}$ and the shape of the IFEDF does not change significantly [see Fig. 7]. Thus, the observed changes in the etch rate cannot be explained by a change in the ion bombardment energy. It is known that both oxygen atom and positive oxygen ion bombardment cause ACL etching. In qualitative agreement with this, we observe a correlation of the etch rate with the measured optical emission from an atomic oxygen line and the computed dissociative electron impact excitation rate shown in Fig. 2(b), which are markers of the atomic oxygen density, and the computed $\mathrm{O}_{2}^{+}$ion flux shown in Fig. 3(b). At small electrode gaps, the atomic oxygen and the positive oxygen ion flux to the wafer are high and, thus, a high etch rate is found. Towards higher gaps, the atomic oxygen flux decreases. The positive oxygen ion flux, however, has a minimum at intermediate gap distances and increases towards higher gaps. Thus, at intermediate electrode gaps, the etch rate is low. At large gaps, the atomic oxygen flux is low, but the ion flux is high. Therefore, a higher etch rate is observed compared to intermediate gap distances, which is, however, lower compared to the smallest gap size, where both the atomic oxygen and the oxygen ion fluxes are high.

We note that the simulation data do not provide a strict proof for the above explanations and these, accordingly, should be understood as hypotheses that warrant further investigations based on a more detailed model.

\section{SUMMARY}

We presented an experimental and computational investigation of the effects of changing the electrode gap on the discharge characteristics and on the etch rate of amorphous carbon layers (ACLs) in low pressure single frequency CCPs operated in oxygen. Experimentally, it was found that, as the electrode gap length is increased, the light emission intensity of an atomic oxygen line decreases gradually and the plasma density increases abruptly. The electron density and the excitation rate for the $844.6 \mathrm{~nm}$ oxygen atomic transition obtained from the particle based numerical simulations of the plasma were found to be in good agreement with the experimental data. The simulations allowed us to obtain a fundamental understanding of the changes of the plasma characteristics as a function of the electrode gap. In particular, a decrease in the mean electron energy and the electronegativity was observed, indicating a change from a plasma with electronegative character at short gaps to a plasma with electropositive character at long gaps. The numerical results also indicated a complex trend of the $\mathrm{O}_{2}^{+}$ion flux to the electrodes as a function of the gap length. These findings were explained by mode transitions of the electron power absorption dynamics from the Drift-Ambipolar mode at low gap distances to the $\alpha$-mode at larger electrode gaps.
The plasma etching of ACLs was also investigated experimentally. It was found that the etch rate of the ACL hard mask depends strongly on the electrode gap distance in a nonmonotonic way. Based on a synergistic combination of the experimental and computational results, this dependence was correlated with the changes in the atomic oxygen density and the $\mathrm{O}_{2}^{+}$ion flux to the electrodes.

\section{ACKNOWLEDGMENTS}

This research was supported by the Korea Research Institute of Standard and Science (KRISS) and the R\&D Convergence Program (1711062007, CAP-17-02-NFRI-01) of National Research Council of Science and Technology (NST) of Republic of Korea. The support of the DFG (German Research Foundation) within the framework of the Sonderforschungsbereich SFBTR 87 (project C1), the US National Science Foundation (Grant No. PHY 1601080), the Hungarian National Office for Research, Development and Innovation (NKFIH) via the Grant Nos. K-119357, PD-121033, and FK-128924, and the J Bolyai Research Fellowship of the HAS is gratefully acknowledged.

\section{REFERENCES}

${ }^{7}$ M. A. Lieberman and A. J. Lichtenberg, Principles of Plasma Discharges and Materials Processing, 2nd ed. (Wiley Interscience, Hoboken, NJ, 2005).

2J. Hopwood, Plasma Sources Sci. Technol. 1, 109 (1992).

${ }^{3}$ H. Conrads and M. Schmidt, Plasma Sources Sci. Technol. 9, 441 (2000).

${ }^{4} \mathrm{~J}$. W. Coburn and H. F. Winters, J. Appl. Phys. 50, 3189 (1979).

${ }^{5}$ H.-C. Lee, Appl. Phys. Rev. 5, 011108 (2018).

${ }^{6}$ R. L. Bates, P. L. S. Thamban, M. Goeckner, and L. J. Overzet, J. Vac. Sci. Technol., A 32, 041302 (2014).

${ }^{7}$ L. J. Overzet and J. T. Verdeyen, Appl. Phys. Lett. 48, 695 (1986)

${ }^{8}$ C. G. N. Lee, K. J. Kanarik, and R. A. Gottscho, J. Phys. D: Appl. Phys. 47, 273001 (2014).

${ }^{9}$ K. J. Kanarik, T. Lill, E. A. Hudson, S. Sriraman, S. Tan, J. Marks, V. Vahedi, and R. A. Gottscho, J. Vac. Sci. Technol. A 33, 020802 (2015).

${ }^{10}$ G. S. Oehrlein and S. Hamaguchi, Plasma Sources Sci. Technol. 27, 023001 (2018).

${ }^{11}$ M. Surendra and D. B. Graves, Appl. Phys. Lett. 59, 2091 (1991).

${ }^{12}$ V. Vahedi, C. K. Birdsall, M. A. Lieberman, G. DiPeso, and T. D. Rognlien, Phys. Fluids B 5, 2719 (1993).

${ }^{13}$ M. J. Colgan, M. Meyyappan, and D. E. Murnick, Plasma Sources Sci. Technol. 3, 181 (1994).

${ }^{14}$ A. Perret, P. Chabert, J.-P. Booth, J. Jolly, J. Guillon, and P. Auvray, Appl. Phys. Lett. 83, 243 (2003).

${ }^{15}$ S. Wilczek, J. Trieschmann, J. Schulze, E. Schuengel, R. P. Brinkmann, A. Derzsi, I. Korolov, Z. Donkó, and T. Mussenbrock, Plasma Sources Sci. Technol. 24, 024002 (2015).

${ }^{16}$ L. D. Tsendin, Phys. Usp. 53, 133 (2010).

${ }^{17}$ M. A. Lieberman, J.-P. Booth, P. Chabert, J. M. Rax, and M. M. Turner, Plasma Sources Sci. Technol. 11, 283 (2002).

${ }^{18}$ V. A. Godyak, R. B. Piejak, and B. M. Alexandrovich, Phys. Rev. Lett. 68, 40 (1992).

${ }^{19}$ V. A. Godyak, R. B. Piejak, and B. M. Alexandrovich, Plasma Sources Sci. Technol. 1, 36 (1992).

${ }^{20}$ P. Belenguer and J. P. Boeuf, Phys. Rev. A 41, 4447 (1990).

${ }^{21}$ J. Schulze, A. Derzsi, K. Dittmann, T. Hemke, J. Meichnser, and Z. Donkó, Phys. Rev. Lett. 107, 275001 (2011).

${ }^{22}$ Y. X. Liu, E. Schuengel, I. Korolov, Z. Donkó, Y. N. Wang, and J. Schulze, Phys. Rev. Lett. 116, 255002 (2016).

${ }^{23}$ Y. X. Liu, E. Schuengel, I. Korolov, Z. Donkó, Y. N. Wang, and J. Schulze, Phys. Plasmas 24, 073512 (2017). 
${ }^{24}$ Y. X. Liu, E. Schuengel, I. Korolov, Z. Donkó, Y. N. Wang, and J. Schulze, Plasma Sources Sci. Technol. 26, 055024 (2017).

${ }^{25}$ T. Lafleur, Plasma Sources Sci. Technol. 25, 013001 (2016).

${ }^{26}$ B. Berger, S. Brandt, J. Franek, E. Schuengel, M. Koepke, T. Mussenbrock, and J. Schulze, J. Appl. Phys. 118, 223302 (2015).

${ }^{27}$ B. Bruneau, T. Lafleur, T. Gans, D. O'Connell, A. Greb, I. Korolov, A. Derzsi, Z. Donkó, S. Brandt, E. Schuengel, J. Schulze, P. Diomede, D. J. Economou, S. Longo, E. Johnson, and J.-P. Booth, Plasma Sources Sci. Technol 25, 01LT02 (2016).

${ }^{28}$ J. Schulze, E. Schüngel, Z. Donkó, and U. Czarnetzki, Plasma Sources Sci. Technol. 19, 045028 (2010).

${ }^{29}$ Y. Zhang, A. Zafar, D. Coumou, S. Shannon, and M. Kushner, J. Appl. Phys. 117, 233302 (2015).

${ }^{30}$ D. J. Coumou, D. H. Clark, T. Kummerer, M. Hopkins, D. Sullivan, and S. Shannon, IEEE Trans Plasma Sci. 42, 1880 (2014).

${ }^{31}$ J. Schulze, E. Schüngel, U. Czarnetzki, M. Gebhardt, R. P. Brinkmann, and T. Mussenbrock, Appl. Phys, Lett. 98, 031501 (2011).

${ }^{32}$ H. C. Lee, M. H. Lee, and C. W. Chung, Appl. Phys. Lett. 96, 071501 (2010).

${ }^{33}$ M. A. Sobolewski and J. H. Kim, J. Appl. Phys. 102, 113302 (2007).

${ }^{34}$ H.-C. Lee and C.-W. Chung, Appl. Phys. Lett. 101, 244104 (2012).

${ }^{35}$ J. Schulze, E. Schuengel, and U. Czarnetzki, Appl. Phys. Lett. 100, 024102 (2012).

${ }^{36}$ H. Lee and C. Chung, Plasma Sources Sci. Technol. 23, 062002 (2014).

${ }^{37}$ H. Lee and C. Chung, Plasma Sources Sci. Technol. 24, 024001 (2015).

${ }^{38}$ D.-Q. Wen, W. Liu, F. Gao, M. A. Lieberman, and Y.-N. Wang, Plasma Sources Sci. Technol. 25, 045009 (2016).

${ }^{39}$ H. C. Lee, S. J. Oh, and C. W. Chung, Plasma Sources Sci. Technol. 21, 035003 (2012).

${ }^{40} \mathrm{M}$. Zaka-ul-Islam, D. O'Connell, W. G. Graham, and T. Gans, Plasma Sources Sci. Technol. 24, 044007 (2015).

${ }^{41}$ B. Berger, T. Steinberger, E. Schuengel, M. Koepke, T. Mussenbrock, P. Awakowicz, and J. Schulze, Appl. Phys. Lett. 111, 201601 (2017).

${ }^{42}$ P. Ahr, E. Schuengel, J. Schulze, T. Tsankov, and U. Czarnetzki, Plasma Sources Sci. Technol. 24, 044006 (2015).

${ }^{43}$ K. Dittmann, D. Drozdov, B. Krames, and J. Meichsner, J. Phys. D: Appl. Phys. 40, 6593 (2007).

${ }^{44}$ K. Matyash, R. Schneider, K. Dittmann, J. Meichsner, F. X. Bronold, and D. Tskhakaya, J. Phys. D: Appl. Phys. 40, 6601 (2007).

${ }^{45}$ C. Kuellig, T. Wegner, and J. Meichsner, Phys. Plasmas 22, 043515 (2015).

${ }^{46}$ D. Sung, V. Volynets, W. Hwang, Y. Sung, S. Lee, M. Choi, and G. Kim, J. Vac. Sci. Technol. A 30, 061301 (2012).

${ }^{47}$ E. Johnson, T. Verbeke, J. Vanel, and J.-P. Booth, J. Phys. D: Appl. Phys. 43, $412001(2010)$

${ }^{48}$ E. Johnson, S. Pouliquen, P. A. Delattre, and J.-P. Booth, J. Non-Cryst. Solids 358, 1974 (2012).

${ }^{49}$ E. C. Neyts, K. Ostrikov, Z. J. Han, S. Kumar, A. C. T. van Duin, and A. Bogaerts, Phys. Rev. Lett. 110, 065501 (2013).

${ }^{50}$ W. Jiang, H. Wang, S. Zhao, and Y. Wang, J. Phys. D: Appl. Phys. 42, 102005 (2009).
${ }^{51}$ S. J. You, S. S. Kim, J. H. Kim, D. J. Seong, Y.-H. Shin, and H. Y. Chang, Appl. Phys. Lett. 91, 221501 (2007).

${ }^{52}$ X. Lu, H. Huang, N. Nemchuk, and R. S. Ruoff, Appl. Phys. Lett. 75, 193 (1999).

${ }^{53}$ N. J. Chou, C. H. Tang, J. Paraszczak, and E. Babich, Appl. Phys. Lett. 46, 31 (1985).

${ }^{54}$ M. A. Hartney, D. W. Hess, and D. S. Soane, J. Vac. Sci. Technol., B: Nanotechnol. Microelectron.: Mater., Process., Meas., Phenom. 7, 1 (1989).

55I. Childres, L. A. Jauregui, J. Tian, and Y. P. Chen, New J. Phys. 13, 025008 (2011).

${ }^{56}$ X. Xue, K. Zhou, J. Cai, Q. Wang, and Z. Wang, Microelectron. Eng. 191, 1 (2018).

${ }^{57}$ A. Derzsi, T. Lafleur, J.-P. Booth, I. Korolov, and Z. Donkó, Plasma Sources Sci. Technol. 25, 015004 (2016).

${ }^{58}$ A. Proto and J. T. Gudmundsson, Plasma Sources Sci. Technol. 27, 074002 (2018).

59J. T. Gudmundsson, D. I. Snorrason, and H. Hannesdottir, Plasma Sources Sci. Technol. 27, 025009 (2018).

60 J. T. Gudmundsson and D. I. Snorrason, J. Appl. Phys. 122, 193302 (2017).

${ }^{61}$ A. Derzsi, B. Bruneau, A. R. Gibson, E. Johnson, D. O'Connell, T. Gans, J.-P. Booth, and Z. Donkó, Plasma Sources Sci. Technol. 26, 034002 (2017).

62J. H. Kim, D. J. Seong, J.-Y. Lim, and K.-H. Chung, Appl. Phys. Lett. 83, 4725 (2003).

${ }^{63}$ J. H. Kim, K.-H. Chung, and Y.-H. Shin, Metrologia 42, 110 (2005).

${ }^{64}$ V. Vahedi and M. Surendra, Comput. Phys. Commun. 87, 179 (1995).

${ }^{65}$ See http://www.lxcat.net for Biagi-v8.9 database (Cross sections extracted from PROGRAM MAGBOLTZ, VERSION 8.9 March 2010); accessed 25 November 2014.

${ }^{66}$ J. T. Gudmundsson, E. Kawamura, and M. A. Lieberman, Plasma Sources Sci. Technol. 22, 035011 (2013).

${ }^{67}$ F. X. Bronold, K. Matyash, D. Tskhakaya, R. Schneider, and H. Fehske, J. Phys. D: Appl. Phys. 40, 6583 (2007).

${ }^{68}$ Z. Donkó, A. Derzsi, I. Korolov, P. Hartmann, S. Brandt, J. Schulze, B. Berger, M. Koepke, B. Bruneau, E. Johnson, T. Lafleur, J.-P. Booth, A. R. Gibson, D. O'Connell, and T. Gans, Plasma Phys. Controlled Fusion 60, 014010 (2018)

${ }^{69}$ A. Greb, K. Niemi, D. O'Connell, and T. Gans, Appl. Phys. Lett. 103, 244101 (2013).

${ }^{70}$ A. Greb, A. R. Gibson, K. Niemi, D. O'Connell, and T. Gans, Plasma Sources Sci. Technol. 24, 044003 (2015).

${ }^{71}$ H. Hannesdottir and J. T. Gudmundsson, Plasma Sources Sci. Technol. 25, 055002 (2016).

${ }^{72}$ B. Horvath, M. Daksha, I. Korolov, A. Derzsi, and J. Schulze, Plasma Sources Sci. Technol. 26, 124001 (2017).

${ }^{73}$ B. Horvath, J. Schulze, Z. Donko, and A. Derzsi, J. Phys. D 51, 355204 (2018).

${ }^{74} \mathrm{R}$. Kollath, Encyclopedia of Physics, edited by S.Fluegge (Berlin, Springer, 1956), Vol. 21, p. 264. 